\title{
Identification of a vertical hopping robot model via harmonic transfer functions
}

Transactions of the Institute of Measurement and Control I-I I

(c) The Author(s) 2015

Reprints and permissions: sagepub.co.uk/journalsPermissions.nav DOI: $10.1177 / 0142331215583327$ tim.sagepub.com

(S)AGE

\author{
İsmail Uyanık', Mustafa M. Ankaralı², Noah J. Cowan², \\ Uluç Saranlı ${ }^{3}$ and Ömer Morgül'
}

\begin{abstract}
A common approach to understanding and controlling robotic legged locomotion is the construction and analysis of simplified mathematical models that capture essential features of locomotor behaviours. However, the representational power of such simple mathematical models is inevitably limited due to the non-linear and complex nature of biological locomotor systems. Attempting to identify and explicitly incorporate key non-linearities into the model is challenging, increases complexity, and decreases the analytic utility of the resulting models. In this paper, we adopt a data-driven approach, with the goal of furnishing an input-output representation of a locomotor system. Our method is based on approximating the hybrid dynamics of a legged locomotion model around its limit cycle as a Linear Time Periodic (LTP) system. Perturbing inputs to the locomotor system with small chirp signals yield the input-output data necessary for the application of LTP system identification techniques, allowing us to estimate harmonic transfer functions (HTFs) associated with the local LTP approximation to the system dynamics around the limit cycle. We compare actual system responses with responses predicted by the HTF, providing evidence that data-driven system identification methods can be used to construct models for locomotor behaviours.
\end{abstract}

\section{Keywords}

System identification, harmonic transfer functions, legged locomotion, periodic systems

\section{Introduction}

Legged morphologies admit a wide range of locomotor behaviours, for which a variety of mathematical models have been proposed. For example, simple spring-mass models, including the Spring-Loaded Inverted Pendulum (SLIP) model (Schwind, 1998), have been very successful in representing running and walking behaviours (Blickhan and Full, 1993; Full and Tu, 1991). The hybrid structure of these models alternates between flight and stance phases, each possessing smooth dynamics with continuous flows; transitions are punctuated with discrete, state-based transitions. Despite the seemingly simple nature of these models, however, their dynamics during stance include non-integrable parts (Holmes, 1990), which prevent the derivation of exact closed-form solutions. Various approximate solutions have been developed in the literature to address this problem (Ankarali and Saranli, 2010; Geyer et al., 2005; Saranli et al., 2010; Schwind and Koditschek, 2000), some of which have also been verified experimentally (Uyanik et al., 2015).

It has also been shown that these models and associated solutions can support the design of hierarchical controllers for more complex platforms and morphologies (Ankarali and Saranli, 2011; Poulakakis and Grizzle, 2007; Saranll, 2002). Moreover, Uyanik et al. (2011) showed that the structure and efficiency of these analytic solutions can also be exploited to yield effective solutions for parameter identification and adaptive control. Nevertheless, such explicit modelling efforts will always suffer from inaccuracies resulting from unmodelled aspects of physical platforms (Uyanik et al., 2015). While one can certainly introduce more complexity along the templateanchors continuum (Full and Koditschek, 1999), beyond a certain point these extended models begin to lose their analytic tractability in order to gain improvements in accuracy.

Here, we propose an alternative approach that steers away from explicit mechanical modelling towards data-driven system identification. Rather than introducing more and more specific, detailed mechanistic features of increasing complexity to mathematical models in order to compensate for particular sources of inaccuracy in a physical platform, such a data-driven approach treats the system as a black box, focusing on the adequacy of available data and the identification method for increased accuracy. The goal with such empirical models is to complement, not replace, mechanistic models

\footnotetext{
'Department of Electrical and Electronics Engineering, Bilkent University, Turkey

${ }^{2}$ Department of Mechanical Engineering, Johns Hopkins University, USA

${ }^{3}$ Department of Computer Engineering, Middle East Technical University, Turkey
}

\section{Corresponding author:}

İsmail Uyanık, Department of Electrical and Electronics Engineering,

Bilkent University, TR-06800 Bilkent, Ankara, Turkey.

Email: uyanik@ee.bilkent.edu.tr 
which have the benefit of trying to explicitly connect system behaviour to physical design details and controller parameters.

More specifically, our approach in this paper is to use a Linear Time Periodic (LTP) system structure to approximately model locomotor behaviours around their limit cycles, using associated system identification techniques to obtain a linearized input-output representation for the system. To this end, we first approximate state-dependent hybrid transitions of these systems as time-dependent transition functions. We then linearize these approximate dynamics around the limit cycle, yielding a piecewise smooth LTP system.

An important and powerful tool for the analysis and datadriven identification of LTP systems is the concept of Harmonic Transfer Functions (HTFs), which are analogous to traditional transfer functions for Linear Time Invariant (LTI) systems (Wereley, 1991). Unlike LTI systems, an input signal with a specific frequency supplied to an LTP system produces output components spread across different harmonics of the periodic system frequency and components of the HTF structure define the coupling between different harmonics. Within this framework, Siddiqi (2001) developed an identification strategy to estimate the individual HTFs for an LTP system by exciting the system using specially designed chirp signals and using modified "power spectral density" and "cross spectral density" functions as in the case of LTI systems. In this work, we adopt this technique to estimate the HTFs for a spring-mass damper model of vertical hopping. Alternative identification strategies for LTP systems were proposed by Hwang (1997) and Louarroudi et al. (2011). In the present study, we rely on chirp signals with their well-defined frequency range and predetermined power spectral density (Annus et al., 2012), rather than the sum of sines inputs used by Louarroudi et al. (2011). Hwang (1997) uses single sine inputs, but the method requires multiple experiments to cover the frequency range that we are interested in this paper. For these reasons, our identification method is based on Siddiqi's approach.

Prior to our work, a system identification method for smooth rhythmic dynamical systems was developed by Kiemel et al. (2013) using continuous-time HTFs. Later, Ankarali and Cowan (2014) developed a new identification method for hybrid (non-smooth) dynamical systems based on discrete-time HTFs. In this paper, we show that identification methods based on continuous-time HTFs can be applied to a clock-driven hybrid dynamical model of locomotion.

In this context, we extend and apply the HTF-based system identification method described in Siddiqi (2001), which we briefly review in the next section, to a clock-driven hybrid vertical locomotion model for which the analytic derivation for the HTF representation appears to be challenging (or infeasible). In so doing, we show that identification methods normally designed for continuous systems can be applied to systems with hybrid components that are inevitable for legged locomotion, while also establishing the accuracy of the identification process through systematic simulation studies.

\section{Harmonic transfer functions}

Many finite-dimensional LTP systems can be described by a state space model of the form

$$
\begin{aligned}
& \dot{\mathbf{x}}(t)=\mathbf{A}(t) \mathbf{x}(t)+\mathbf{B}(t) \mathbf{u}(t) \\
& \mathbf{y}(t)=\mathbf{C}(t) \mathbf{x}(t)+\mathbf{D}(t) \mathbf{u}(t)
\end{aligned}
$$

where $\mathbf{A}(t), \mathbf{B}(t), \mathbf{C}(t)$, and $\mathbf{D}(t)$ are all periodic with period $T$. Despite its linear nature, the time dependence of matrices in this representation makes it impossible to directly apply analysis and identification techniques developed for LTI systems.

System identification methods for asymptotically stable LTI systems are well established, thanks in large measure to the one-to-one mapping between frequency response characteristics of input and output signals at steady state. This allows one to obtain empirical frequency response functions (i.e. "Bode plots") describing the magnitude and phase changes in the input signal at each specific frequency for the desired system. Due to the time dependence of matrices in equation (1), however, the response to a sinusoidal input with a specific frequency passing through an LTP system may include multiple (possibly infinite) harmonics, with different magnitudes and phases. In general, neglecting all higher order harmonics of the system (Leonhard, 1963) to obtain a one-toone mapping between the input and output signals in the frequency domain may lead to unacceptable inaccuracies. Consequently, a different approach is required for datadriven identification of such systems.

Wereley and Hall (1990) proposed a solution to this problem by transforming the input and output signals to exponentially modulated periodic (EMP) signals. In this domain, it is possible to obtain a one-to-one mapping between the Fourier coefficients of the input and output EMP signals. In the resulting structure input-output representation is determined by multiple (possibly infinite) parallel LTI sub-systems whose inputs are multiplied by complex periodic signals. Figure 1 illustrates the resulting HTF structure (original source Möllerstedt, 2000). These LTI sub-systems are called HTFs and they characterize the frequency response characteristics of an LTP system. A detailed frequency domain analysis of LTP systems based on HTFs has also been investigated by Sandberg et al. (2005). Subsequent sections in this paper review the theoretical derivations behind the HTF framework as proposed by Möllerstedt (2000). We then present a datadriven identification strategy (Siddiqi, 2001) to estimate these HTFs in the absence of an explicitly constructed state space representation of the system.

\section{Structure of harmonic transfer functions}

In this section, we summarize the derivation of the HTF structure using the time periodic impulse response representation of an LTP system (Möllerstedt, 2000) for reader convenience and to establish notation. The output of an LTP system, such as those in the form of equation (1), can also be represented using its time periodic impulse response functions as

$$
y(t)=\int_{0}^{t} H(t, \tau) u(\tau) \mathrm{d} \tau
$$

where $H(t, \tau)=H(t-T, \tau-T)$ and $T$ is the period of the system. In other words, all time-varying impulse response 


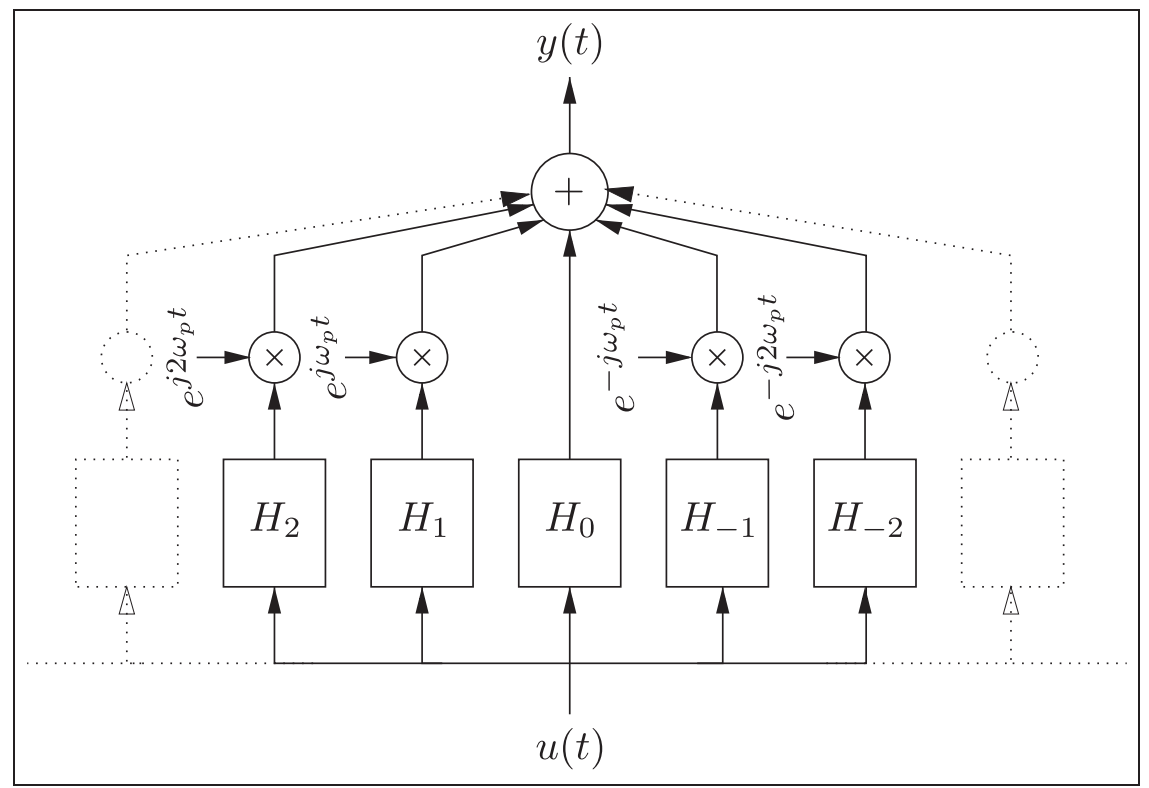

Figure I. Illustration of the HTF structure (adapted from Möllerstedt, 2000). The input-output relation of an LTP system can be expressed by multiple (possibly infinite) parallel LTI sub-systems.

functions of the system are periodic in both arguments. Letting $\tau=t-r$, we have $H(t, \tau)=H(t, t-r)$ which is $T$ periodic in $t$. This periodicity allows us to expand $H(t, t-r)$ through an infinite Fourier series expansion with pumping frequency, $\omega_{\mathrm{p}}=2 \pi / T$, yielding

$$
\begin{aligned}
H(t, t-r) & =\sum_{k=-\infty}^{\infty} H_{k}(r) e^{j k \omega_{\mathrm{p}} t}, \\
H_{k}(r) & :=\frac{1}{T} \int_{0}^{\mathrm{T}} e^{-j k \omega_{\mathrm{p}} t} H(t, t-r) \mathrm{d} t
\end{aligned}
$$

Switching back to $\tau$ through $r=t-\tau$ gives

$$
H(t, \tau)=\sum_{k=-\infty}^{\infty} H_{k}(t-\tau) e^{j k \omega_{\mathrm{p}} t}
$$

Substituting equation (4) into equation (2), we get

$$
\begin{aligned}
y(t) & =\sum_{k=-\infty}^{\infty} \int_{0}^{\mathrm{T}} H_{k}(t-\tau) e^{j k \omega_{\mathrm{p}} t} u(\tau) \mathrm{d} \tau \\
& =\sum_{k=-\infty}^{\infty} \int_{0}^{\mathrm{T}} H_{k}(t-\tau) e^{j k \omega_{\mathrm{p}} t-\tau} u(\tau) e^{j k \omega_{\mathrm{p}} \tau} \mathrm{d} \tau \\
& =\sum_{k=-\infty}^{\infty}\left(H_{k}(t) e^{j k \omega_{\mathrm{p}} t}\right) *\left(u(t) e^{j k \omega_{\mathrm{p}} t}\right)
\end{aligned}
$$

where * denotes the convolution operator. Finally, taking the Laplace transform of both sides yields

$$
Y(s)=\sum_{k=-\infty}^{\infty} H_{k}\left(s-j k \omega_{\mathrm{p}}\right) U\left(s-j k \omega_{\mathrm{p}}\right)
$$

Now, let us define $G_{k}(s):=H_{k}\left(s-j k \omega_{\mathrm{p}}\right)$ as the elements of the HTF structure. An alternative method to obtain these
HTF components relies on a time-varying state space representation and the principle of Harmonic Balance (Wereley, 1991; Wereley and Hall, 1990).

\section{Non-parametric identification of harmonic transfer functions}

When an explicit representation of a system is given, either in state space form or through an impulse response function as in equation (2), the derivations of the previous section can be used to obtain the corresponding HTFs. However, manual construction of such explicit models is often impractical beyond a certain level of complexity. Consequently, the estimation of HTFs without the need for such explicit models is of great practical interest. In this section, we review the datadriven system identification method introduced by Siddiqi (2001) for LTP systems, together with our extensions to support its application for clock-driven legged locomotion models.

The HTF structure of equation (6) includes an infinite number of harmonics, which is problematic for practical applications. Consequently, these harmonic components are often truncated beyond a certain order to enable effective computational implementations. Similarly, some LTP system identification methods also focus on a preselected number of harmonics. For clarity, our review of Siddiqi's method in this section considers only three HTFs, $\hat{G}_{0}, \hat{G}_{-1}$ and $\hat{G}_{1}$, leading to a representation of the system output in the frequency domain as

$$
\begin{aligned}
Y(j \omega) \approx \hat{Y}(j \omega):= & \hat{G}_{0}(j \omega) U(j \omega)+\hat{G}_{-1}(j \omega) U\left(j \omega+j \omega_{\mathrm{p}}\right) \\
& +\hat{G}_{1}(j \omega) U\left(j \omega-j \omega_{\mathrm{p}}\right)
\end{aligned}
$$




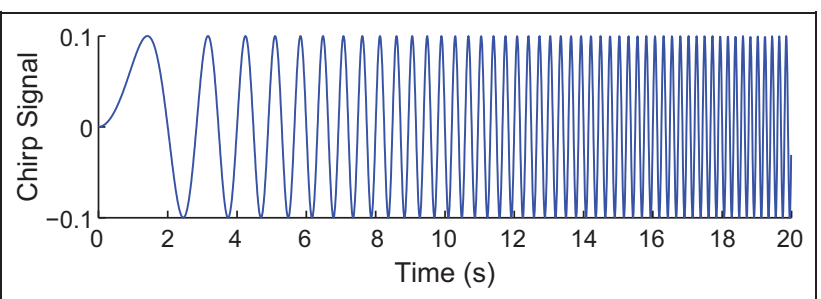

Figure 2. Chirp signal used to perturb the system for system identification, consisting of a sinusoid with amplitude 0.1 and frequency increasing linearly in time within the range $(0,5] \mathrm{Hz}$ in $20 \mathrm{~s}$.

where variables annotated with a hat denote estimated versions of their system counterparts. Based on this definition, the data-driven system identification problem can be reduced to the problem of estimating the quantities $\hat{G}_{0}(j \omega), \hat{G}_{-1}(j \omega)$ and $\hat{G}_{1}(j \omega)$ at each specific frequency, $\omega$, such that the difference between the measured and estimated output vectors is minimized.

The correct choice of input signals plays a crucial role in the system identification process. Input signals must be designed to expose as much dynamic behaviour in the system as possible. To this end, chirp signal inputs as shown in Figure 2, can be used to to cover a sufficiently wide frequency spectrum. The accuracy of the identification critically depends on particular aspects of these chirp inputs, such as their duration, frequency range and sweep rate. Moreover, the phase timing of the input signal relative to the LTP system also effects the activation of different dynamic components within the system. Siddiqi (2001) addresses these problems by designing a single input sequence, incorporating phase-shifted replicas of an original chirp signal spanning a sufficiently wide range of frequencies. Phase shifts ensure that the system is excited evenly throughout the system's period, while the wide frequency range explores different modes in the system towards a complete characterization of the effects of internal system dynamics on system output.

Using the resulting input-output pairs, one can then compute the "extended power spectral density" and "extended cross spectral density" functions $\Phi_{\mathbf{U U}}(\omega)$ and $\Phi_{\mathbf{U Y}}(\omega)$, respectively. Even though $\Phi_{\mathbf{U U}}(\omega)$ and $\Phi_{\mathbf{U Y}}(\omega)$ are analogous to power spectral density and cross spectral density functions for LTI systems, they differ in their representation as matrix operators. Detailed definitions of these functions can be found in Siddiqi (2001) and are omitted here for space considerations.

Now, the three HTF components of the LTP system can be estimated as

$$
\hat{G}(j \omega):=\left[\begin{array}{c}
\hat{G}_{1} \\
\hat{G}_{0} \\
\hat{G}_{-1}
\end{array}\right]=\left(\Phi_{\mathbf{U U}}\right)^{-1} \Phi_{\mathbf{U Y}}
$$

An important problem with this formulation, however, is that it embodies an under-determined fitting problem. A single pair of input and output vectors may not be sufficient to accurately estimate all three HTFs even though equation (7) represents a single-input single-output LTP system. In order to address this problem, Siddiqi (2001) observes continuity properties of physical transfer functions and introduces additional constraints to penalize high curvature within each HTF towards better output prediction performance. More formally, having modelled the system with three HTFs, its output response due to an input can be expressed as

$$
\begin{aligned}
Y(j \omega) & =\sum_{k=-1}^{1} U\left(j \omega-k j \omega_{\mathrm{p}}\right) \hat{G}_{k}+E(j \omega) \\
& =\hat{Y}(j \omega)+E(j \omega)
\end{aligned}
$$

The error term captures the difference between the measured system response and the predictions of the estimated HTFs. The cost function adopted by Siddiqi (2001) for the minimization problem penalizes the quadratic output prediction error and the curvature of the HTFs, taking the form

$$
\mathbf{J}:=\left[\left(\mathbf{Y}-\mathbf{U}^{\mathrm{T}} \hat{\mathbf{G}}\right)^{2}+\alpha\left(\mathbf{D}^{2} \hat{\mathbf{G}}\right)^{2}\right]
$$

where $\mathbf{D}^{2}$ is the second-order difference operator, $\alpha$ is a scalar weight to tune the smoothness of the resulting transfer functions, and $\mathbf{Y}, \mathbf{U}$ and $\widehat{\mathbf{G}}$ are defined as in Siddiqi (2001). Differentiating $\mathbf{J}$ with respect to $\hat{G}$ and equating to zero yields the estimated HTFs as

$$
\hat{G}(j \omega)=\left(\Phi_{\mathbf{U U}}+\alpha \mathbf{D}^{4}\right)^{-1} \Phi_{\mathbf{U Y}}
$$

\section{Identification of a vertical hopping robot model}

In this section, we apply the HTF system identification method described in the previous section to a vertical hopping robot model, VHOP, that captures some aspects of the wellknown SLIP model of running. We also provide simulation results to illustrate our approach and to characterize the performance of the system identification method applied to the VHOP system. Note that all the system simulations including the estimation of HTFs are implemented in a Matlab environment by using standard ordinary differential equation solvers and built-in matrix operations.

\section{The vertical hopping model}

The VHOP model, illustrated in Figure 3 consists of a vertically constrained point mass attached to a compliant leg with viscous damping. A linear actuator in parallel with the leg spring is incorporated to compensate for energy losses due to damping, and to implement behavioural controllers. The model also incorporates a very small mass at the toe which also allows both better correspondence to physical robot platforms, as well as the ability to apply inputs to the system during flight.

Our use of a linear actuator as an input to the system is inspired by similarly structured robot implementations in the literature (Piovan and Byl, 2013; Secer and Saranli, 2013). There are two significant roles for the linear actuator in the VHOP model. First, it allows us to obtain a clock-driven 


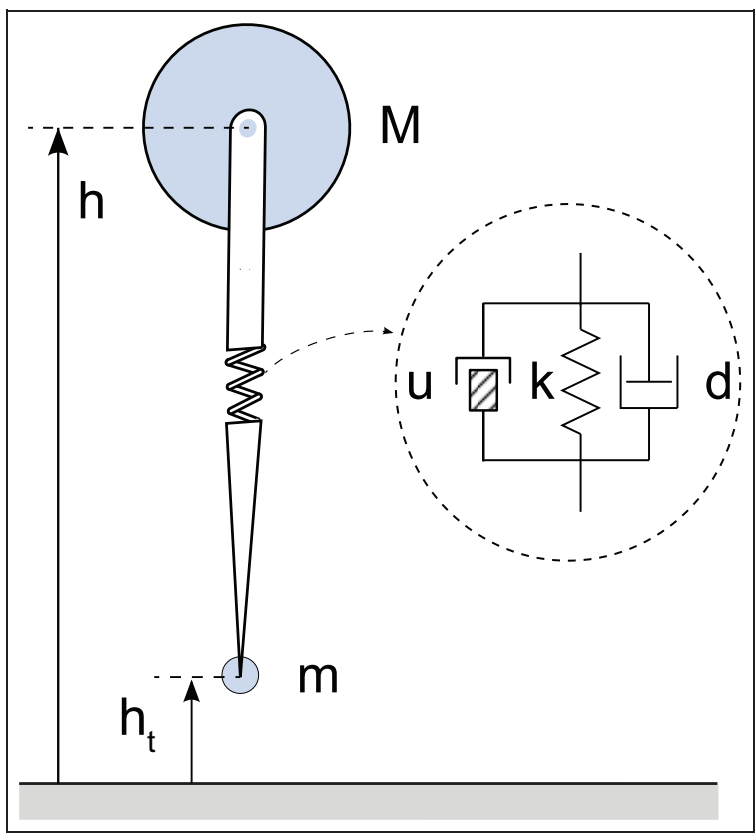

Figure 3. The VHOP model with leg compliance, damping and a parallel linear actuator.

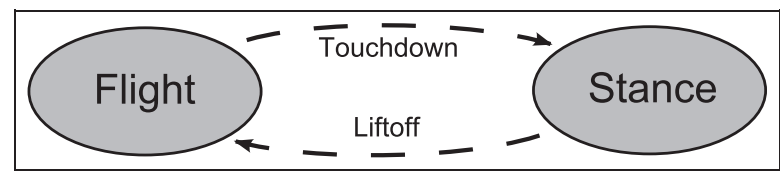

Figure 4. The two phases of locomotion with the VHOP model and associated transition events. Each phase has its own smooth flow.

structure, facilitating the construction of asymptotically stable limit cycles. Second, it can also be used to inject additional 'external' input signals to support our system identification approach. This clock-driven structure with an additive input allows us to avoid phase resetting (Kiemel et al., 2013) and the complications associated with estimating phase (Revzen and Guckenheimer, 2008). Previously, Sracic and Allen (2011) also adopted clock-driven models to perform LTP type analysis on non-linear systems.

Our ultimate goal is to apply the techniques in this paper to more complex models such as the SLIP and its many variants. As a first step, however, we use the relatively simple VHOP model to keep our focus on LTP system identification of hybrid dynamics. Non-linearities and control system challenges associated with more complex models are hence left out of the scope of the present study. Nevertheless, despite its simplicity, the VHOP model still possesses some of the critical features of locomotor dynamics, including hybrid dynamics with flight and stance phases, discontinuities in the state due to collisions, as well as periodic behaviour in the form of limit cycles.

The VHOP model alternates between stance and flight phases and hence can be modelled as a hybrid dynamical system consisting of a set of smooth flows with discrete transitions between them (Guckenheimer and Johnson, 1995). As usual, the stance phase corresponds to states where the foot is in contact with the ground, while the flight phase corresponds to states when the robot is off the ground Transitions from and to stance are called "liftoff" and "touchdown" events, respectively. A simple diagram of VHOP phases and transitions is shown in Figure 4. The system state for both phases is defined as

$$
\mathbf{x}:=\left[\begin{array}{llll}
h & h_{t} & \dot{h} & \dot{h}_{t}
\end{array}\right]^{\mathrm{T}}
$$

\section{Vertical hopping system dynamics}

Structural differences between the stance and flight phases require their modelling through separate differential equations, leading to the hybrid nature of the VHOP model. During flight, the toe has no contact with the ground and is hence free to move vertically, leading to the equations of motion

$$
\begin{gathered}
M \ddot{h}=-M g-d\left(\dot{h}-\dot{h}_{t}\right)+k\left(\ell_{0}-\left(h-h_{t}\right)\right)+u(t) \\
m \ddot{h}_{t}=-m g+d\left(\dot{h}-\dot{h}_{t}\right)-k\left(\ell_{0}-\left(h-h_{t}\right)\right)-u(t)
\end{gathered}
$$

where the viscoelastic leg has damping coefficient, $d$, spring constant, $k$, and rest length $\ell_{0}$. Even though this formulation leaves the actuator input $u(t)$ unspecified, we will impose a clock-driven structure on this input signal of the form $u(t)=a$ $\cos \left(\omega_{\mathrm{p}} t\right)+u_{\mathrm{c}}(t)$, incorporating a periodic open-loop forcing term to achieve a limit cycle, and an additive $u_{\mathrm{c}}(t)$ for additional control affordance. The signal $u_{\mathrm{c}}(t)$ will be the input used for system identification.

During stance, the toe is assumed to remain stationary on the ground until liftoff and the body mass experiences the spring, damper and actuator forces. The corresponding equations of motion hence take the form

$$
\begin{gathered}
M \ddot{h}=-M g-d \dot{h}+k\left(\ell_{0}-h\right)+u(t) \\
m \ddot{h}_{t}=0
\end{gathered}
$$

where the same actuator action is used. Kinematic and dynamic parameters for both phases are detailed in Table 1, chosen to be consistent with the physical monopod platform in our laboratory (Uyanik, 2011) for future extensions to real-world applications.

The final remaining components in the hybrid dynamics are the threshold and transition functions. The liftoff event occurs during stance when the net vertical force on the toe mass crosses zero, beyond which the toe lifts off the ground and the flight phase starts. The corresponding boundary condition is $f_{\mathrm{lo}}(\mathbf{x}):=-d \dot{h}+k\left(\ell_{0}-h\right)+a \cos \left(\omega_{\mathrm{p}} t\right)+u_{\mathrm{c}}(t)=0$. In contrast, the touchdown event, defining the transition from flight to stance, occurs when the toe touches the ground, captured through the boundary condition $f_{\mathrm{td}}(\mathbf{x}):=h_{t}=0$.

System trajectories remain continuous through the liftoff event, but the touchdown event induces discontinuous state trajectories due to the associated collision, modelled through a transition function ensuring that $\dot{h}_{t}\left(t_{\mathrm{td}}^{+}\right)=0$. An important consequence of this discrete change is that the derivation of a 
Table I. VHOP model parameters.

\begin{tabular}{llll}
\hline Parameter & Description & Value & Unit \\
\hline$M$ & Body mass & 2.7 & $\mathrm{~kg}$ \\
$m$ & Toe mass & 0.05 & $\mathrm{~kg}$ \\
$k$ & Compliance & 6500 & $\mathrm{~N} / \mathrm{m}$ \\
$d$ & Damping & 12 & $\mathrm{Ns} / \mathrm{m}$ \\
$a$ & Pumping magnitude & 75 & $\mathrm{~N}$ \\
$\omega_{\mathrm{P}}$ & Pumping frequency & $2 \pi / 0.33$ & $\mathrm{rad} / \mathrm{s}$ \\
$\mathrm{g}$ & Gravity & $9.8 \mathrm{I}$ & $\mathrm{m} / \mathrm{s}^{2}$ \\
$\ell_{0}$ & Rest length & 0.2 & $\mathrm{~m}$ \\
\hline
\end{tabular}

closed-form, time-varying state space model is not feasible with available methods. However, Ankarali and Cowan (2014) explicitly showed that a hybrid dynamical system with discrete jumps in system states and even system dimension can be modelled (locally) with a discrete-time impulse response function by choosing a set of Poincaré sections and considering the mapping between those sections. Motivated by this result, we conjecture that the discrete jumps and hybrid transitions can be embedded into a continuous-time, time-periodic impulse response function model. This is more general than a state space model and hence allows us to utilize the HTF structure. We leave attempts to prove this conjecture as a future work. Note, however, that analytic derivations of timeperiodic impulse response functions are not straightforward even for very simple LTP systems, which further motivates our use of a data-driven system identification approach.

\section{Non-parametric system identification for the vertical hopping model}

The VHOP dynamics of equations (14) and (15) clearly do not correspond to a linear time-periodic system. Linearization of these dynamics around an isolated point is also not useful since the expected behaviour takes the form of periodic trajectories that never stabilize around a single point in the state space. Consequently, our approach is to linearize the system around its periodic behaviour.

We start by assuming that the system has an asymptotically stable limit cycle $\mathbf{x}_{\mathrm{lc}}(t):=\left[h_{\mathrm{lc}}(t), h_{t, \mathrm{lc}}(t), \dot{h}_{\mathrm{lc}}(t), \dot{h}_{t, \mathrm{lc}}(t)\right]^{\mathrm{T}}$ with period $T$ when $u_{\mathrm{c}}(t)=0$. Such a limit cycle can be obtained through suitable choices of the periodic excitation component $a \cos \left(\omega_{\mathrm{p}} t\right)$ within $u(t)$. For example, choosing $a=75$ and $\omega_{\mathrm{p}}=2 \pi / 0.33$, results in the limit cycle illustrated in Figure 5 that appears to be asymptotically stable according to numerical simulations. In this paper we selected the height of the upper mass, $h(t)$, as our output measurement to construct an input-output model. We treat the output relative to the nominal behaviour on the limit cycle, i.e. $y(t):=h(t)-h_{\mathrm{lc}}$ $(t)$, where $h_{\mathrm{lc}}(t)$ is the robot height on the limit cycle, assumed to be known (e.g. recorded during operation with $u_{\mathrm{c}}(t)=0$ ). Then, the resulting LTP system can be modelled via its impulse response

$$
y(t)=\int_{0}^{t} H(t, \tau) u_{\mathrm{c}}(\tau) \mathrm{d} \tau
$$

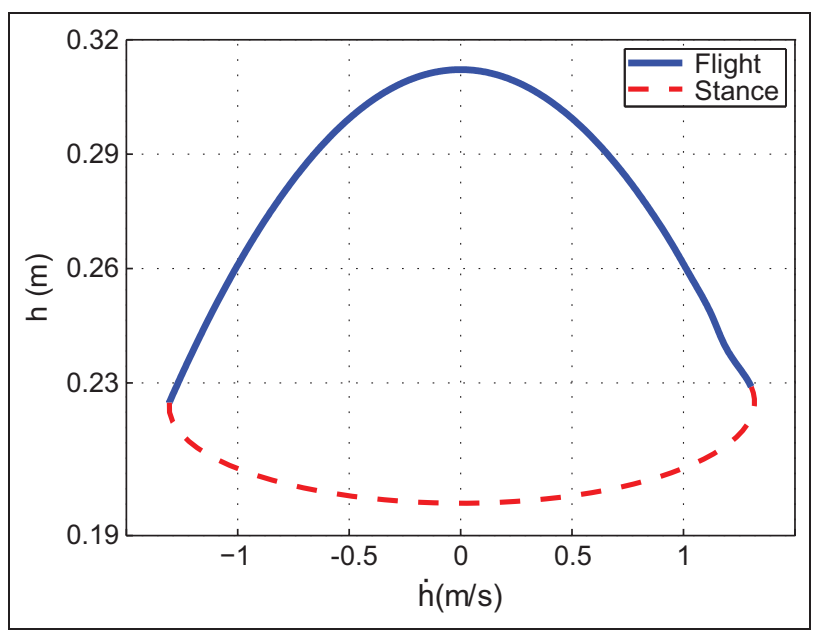

Figure 5. A cross-section of an example VHOP limit cycle obtained with the periodic excitation $u(t)=75 \cos (2 \pi t / 0.33)$. Red and blue sections represent the stance and flight phases on the limit cycle, respectively.

where the impulse response function is periodic, $H(t, \tau)=$ $H(t-T, \tau-T)$, with period $T=2 \pi / w_{\mathrm{p}}$. We apply the system identification method described earlier on these coordinates. To this end, we use $u_{\mathrm{c}}(t)$ to perturb the system away from the limit cycle and analyze the effects on system trajectories. As noted earlier, chirp signals are a good choice for these perturbations, exciting as many modes and components in the system dynamics as possible.

In particular, we use an input signal formed by the concatenation of 21 phase-shifted instances of the chirp signal illustrated in Figure 2, whose frequency changes linearly in the range of zero to $5 \mathrm{~Hz}$ in $20 \mathrm{~s}$. Each instance of this chirp signal is shifted in time by $T / 21$ relative to the previous signal. The magnitude of these chirp signals was chosen through manual tuning in such a way that the perturbations are sufficient, but do not appear to excite significant non-linearities. Each chirp signal $u_{\mathrm{c}}^{i}\left(t_{i}\right)$ with $i \in\{1,2, \ldots, 21\}$, is hence defined as

$$
u_{c}^{i}\left(t_{i}\right)=0.1 \sin \left(0.25 * \pi * t^{2}\right)
$$

where $t_{i}:=t-((i-1) D-(i-1) T / 21) \in[0,20)$ and $D=20 \mathrm{~s}$ is the duration of each chirp application. This yields the final form of our perturbation input (partially shown in Figure 6) as

$$
u_{\mathrm{c}}(t)=\left\{\begin{array}{cl}
u_{\mathrm{c}}^{1}\left(t_{1}\right), & \text { if } 0 \leq t<D \\
\vdots & \vdots \\
u_{\mathrm{c}}^{21}\left(t_{21}\right), & \text { if } 20 D \leq t<21 D \\
0, & \text { if } 21 D \leq t
\end{array}\right.
$$

Finally, we compute the Fourier transforms of the input and output signals as $u_{\mathrm{c}}(j w)$ and $Y(j w)$, respectively. The identification methods described earlier compare the actual output to the predicted output $\hat{Y}(j w)$ of equation (7), resulting in the desired estimates of the three HTFs $\hat{G}_{0}, \hat{G}_{-1}$ and $\hat{G}_{1}$ for the VHOP system. 


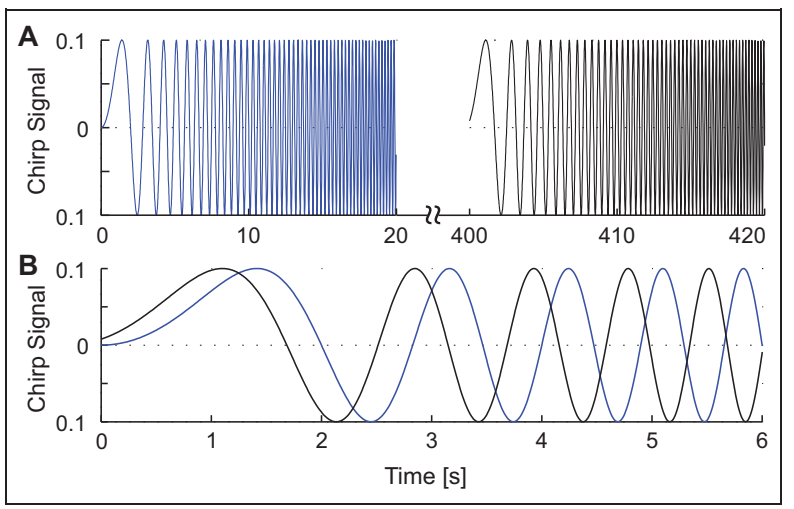

Figure 6. The perturbation input $u_{c}(t)$ used for the system identification process. (A) Phase-shifted repetitions of the original chirp signal, concatenated sequentially (only the $I^{\text {st }}$ and $21^{\text {st }}$ are shown for better illustration). (B) $I^{\text {st }}$ and $2 I^{\text {st }}$ chirp signals superimposed on top of each other for better visualization of phase difference between them.

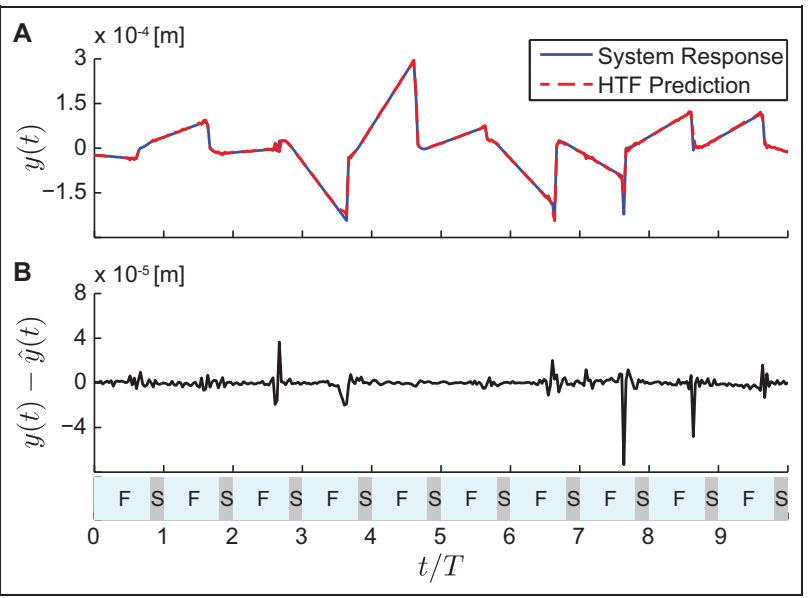

Figure 7. Prediction performance of HTFs with the chirp input training signal. (A) The VHOP system output, (B) discrepancy between the actual and predicted system outputs. The time axis is normalized with the hopping period. Stance and flight phases are indicated at the bottom by the letters $S$ and $F$, respectively.

We evaluate the accuracy of the system identification by comparing the output from VHOP dynamics to the inverse Fourier transform of $\hat{Y}(j w)$ obtained using the estimated HTFs in $\hat{G}$. Figure 7(A) shows the output $y(t)$ for the VHOP response to the chirp signal defined by equation (5), whereas Figure 7(B) shows the discrepancy between the actual and predicted system outputs as a function of time.

As seen in Figure 7, the estimated harmonic transfer functions can successfully predict system response. This result, however, is for the response of the system to the chirp input used for system identification itself and hence is not suitable for a fair evaluation of the prediction performance. A good predictor should be able to estimate system outputs for input signals that might differ substantially from those used for system identification. In the following section, we will present a more systematic characterization of the predictive accuracy obtained through the estimated HTFs using sinusoid and step inputs.

\section{Prediction accuracy of HTF responses to sinusoid and step inputs}

To evaluate the prediction performance of the HTFs, we validate the input-output model identified using chirp excitation on sinusoidal and step input waveforms. We calculate the percentage error based on the difference between the actual and predicted system responses to a particular input

$$
E_{\mathrm{rms}}:=100 \frac{\sqrt{\frac{1}{T_{\mathrm{rms}}} \int_{0}^{T_{\mathrm{rms}}}(y(t)-\hat{y}(t))^{2} \mathrm{~d} t}}{\sqrt{\frac{1}{T_{\mathrm{rms}}} \int_{0}^{T_{\mathrm{rms}}} y(t)^{2} \mathrm{~d} t}}
$$

where $T_{\mathrm{rms}}$ is the duration of the sinusoidal input.

We simulate the VHOP dynamics of the previous section using sinusoidal inputs with amplitude 0.1 and frequencies in the range $f \in[0.1,20] \mathrm{Hz}$ to find the 'actual' outputs of the system. We then compute the output predictions by plugging the previously estimated HTFs into the extended version of equation (7) with $n_{h}$ harmonics for comparison.

Figure 8(A) illustrates $E_{\text {rms }}$ for each input sinusoid frequency when the system identification was performed with a chirp signal covering frequencies from 0 to $5 \mathrm{~Hz}$, whereas Figure 8(B) illustrates the same quantity when system identification was performed with a wider chirp signal covering frequencies from 0 to $10 \mathrm{~Hz}$. Our results show that increasing the frequency coverage of the chirp signal used for training increases the accuracy of the resulting HTF representation for higher frequencies. This is, of course, expected since exciting the system with a wider frequency range allows the HTFs to be properly trained to also match the system response for these higher frequencies. Both the smoothness condition imposed by the system identification process, as well as the nature of LTP systems wherein an input with a single frequency component yields many other frequencies in the output, result in improvements in prediction accuracy for even higher frequency ranges when the chirp spectrum is increased. These results show that the choice of training input has a substantial impact on the accuracy of the resulting HTF estimates.

As noted, practical implementation of this system identification method requires truncating the HTF beyond a certain order, but this threshold cannot be determined beforehand, in general. Consequently, we have explored the effect of incorporating different numbers of HTFs on prediction performance. The four plots in each graph of Figure 8 correspond to system identification with $11,15,21$ and 31 HTFs taken into account. Theoretically, we should expect prediction accuracy to increase when more harmonics are included in the representation, which we verify empirically as illustrated in Figure 8. Beyond a certain number of harmonics (21 in our case), improvements no longer seem substantial, suggesting a reasonable threshold for our system. Including additional harmonics only increases computational complexity without any further significant improvements in accuracy. 


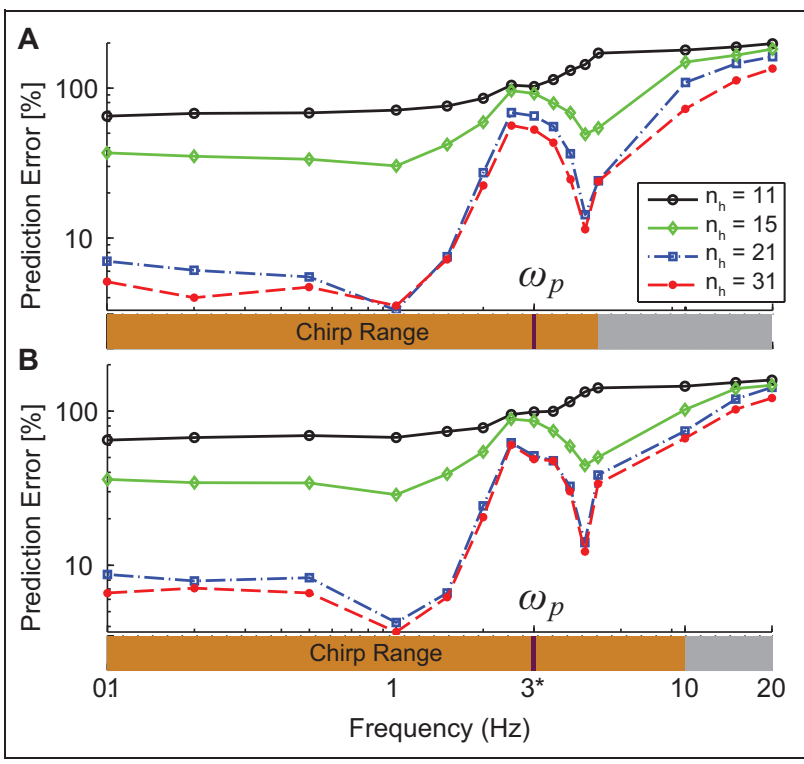

Figure 8. Percentage output prediction errors $E_{\text {rms }}$ for the HTF representation of the VHOP system in response to single sinusoid excitations at different frequencies in the range $[0,20] \mathrm{Hz}$. (A) The HTF representations obtained through training with a chirp signal spanning frequencies in the range 0 to $5 \mathrm{~Hz}$. (B) HTF representations obtained using a chirp input from 0 to $10 \mathrm{~Hz}$.

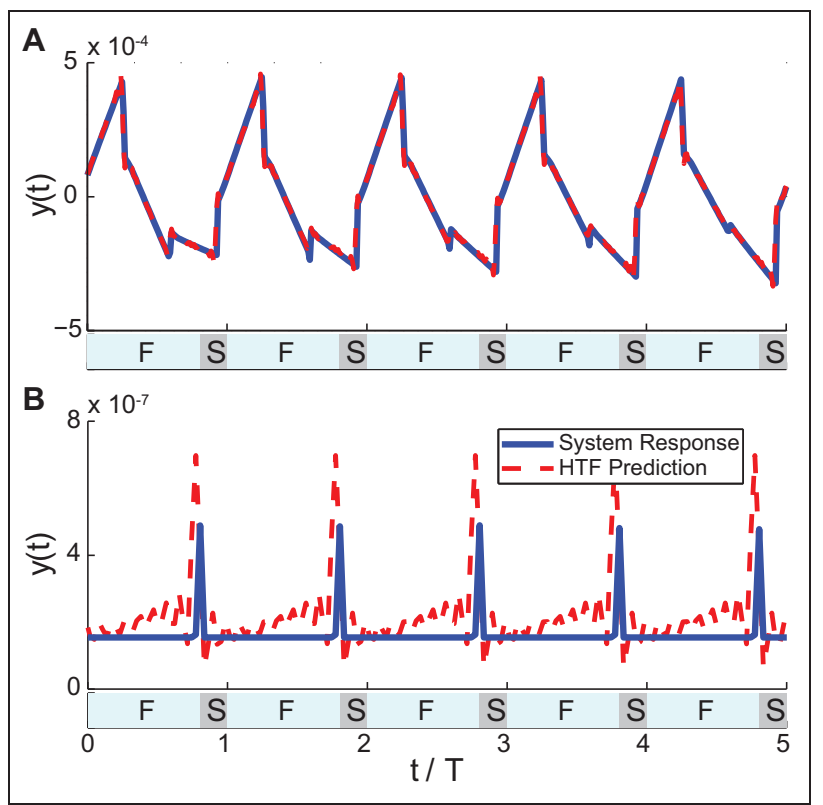

Figure 9. Prediction performance of HTFs with the test input signals. Stance and flight phases indicated by $\mathrm{S}$ and $\mathrm{F}$, respectively. Comparison between measured and predicted system responses for two types of inputs (not used for training): (A) I Hz sinusoidal input and (B) step input. In both cases, steady-state response is shown.

A final comment is required on the performance results of Figure 8 . The predictive performance of the HTF degrades significantly around $3 \mathrm{~Hz}$, which corresponds to the VHOP limit cycle frequency. As detailed before, LTP systems generate output signals at the input frequency plus harmonics of the pumping frequency. When the input and system frequencies are same ( $\omega_{\mathrm{p}}=3 \mathrm{~Hz}$ in our case), different harmonics at multiples of $\omega_{\mathrm{p}}$ may also affect the response at this frequency. Ankarali and Cowan (2014) showed that the identification of HTF should not be performed at frequencies $k \frac{\omega_{\mathrm{p}}}{2}, k \in \mathbb{Z}$ when using sum-of-sines input stimuli for identification. A similar phenomena may be contributing to the errors in our result. However, it is also possible that our linearity assumption is being violated near the pumping frequency. Addressing this problem further is left to future work

In addition to sinusoid inputs at specific frequencies, we have also investigated HTF prediction performance under a step input with magnitude 0.01 . Note, once again, that system identification is still performed with a chirp signal and the step input is only applied for characterizing the prediction performance of the resulting HTF representation. The step input was applied to the system after the $200^{\text {th }}$ cycle, making sure that it has reached steady state. In this case, the estimated system output was computed using an extended version of equation (7) with $n_{h}$ harmonics identified using the chirp signal. Then inverse Fourier transform was used to compute the time domain step response prediction of the HTF system.

Figure 9 illustrates a comparison of measured and predicted responses of the HTF system for a single sinusoid at frequency $1 \mathrm{~Hz}$ in panel (A) and the step input in panel (B). As expected, the HTF representation accurately predicts system response as shown in Figure 9(A), consistent with the results shown in Figure $8\left(E_{\mathrm{rms}}=3.3 \%\right)$. On the other hand, the prediction results for the step input exhibit significant errors as shown in Figure 9(B). Even though the HTF response captures the qualitative behaviour of the step response, including the spiked response around touchdown, noticeable errors remain with $E_{\mathrm{rms}}=68 \%$. This is somewhat similar to the large prediction errors observed around the system periodic frequency and may be due to the harmonics of the step input in the pumping frequency.

One possible reason for these errors may be the relatively small range of frequencies covered by the chirp signal for the identification process. Unfortunately, increasing the frequency range covered by the chirp signal requires substantially longer durations for the input signal. This dramatically increases both the duration of the simulation for data collection, as well as the computational complexity of the identification process. Even though increasing the frequency range, and hence the chirp duration, may increase accuracy, it would impair the feasibility of the system identification method on physical robot platforms due to the resulting need for substantially longer experiments with sustained locomotion.

The non-linearities and the hybrid nature of the VHOP model may also be contributing to these errors. Note that both the system identification and evaluation tests were performed around a limit cycle of the VHOP model. Our method assumes that trajectories around the limit cycle exhibit hybrid transitions that are consistently timed with the limit cycle. However, especially for trajectories obtained with a step input, these transitions may occur much earlier than the limit cycle's corresponding transition. This violates one of the 
underlying assumptions in representing system behaviour around the limit cycle as an LTP system. Moreover, the unidirectional forcing of the step input results in a larger deviation from the limit cycle than the symmetric sinusoid input, which may lead to a further violation of the linearity assumption behind the LTP representation. These effects can be more significant around the harmonics of the pumping frequency, which also includes the DC frequency.

Despite these prediction errors, however, an important feature observed in Figure 9(B), is the ability of the HTF representation to predict the hybrid transitions in the system output resulting from the collision at touchdown. The effects of such impact collisions were investigated in a number of studies (Hutter et al., 2010; Uyanik et al., 2015), and are rather difficult to model within explicitly constructed models. Consequently, the ability of a data-driven system identification approach to model such hybrid features of a system is promising since they are an inevitable part of any locomotor system.

\section{Prediction accuracy of HTF responses under uncorrelated input and output noise}

In the previous section, we investigated the prediction performance of the estimated HTFs for different input waveforms assuming perfect measurement of input and output signals. However, our goal is to develop a data-driven system identification framework applicable to physical legged robot platforms. Therefore, we contaminate the input-output data used for system identification with zero-mean Gaussian noise in order to simulate its performance in more realistic settings.

In order to accomplish this, we use the noise modelling approach for HTFs adopted by Hwang (1997). Figure 10 is a block diagram representation of how noise affects the system identification data. Measured input and output data are corrupted by uncorrelated noise with zero mean and standard deviations; $\sigma_{U}$ and $\sigma_{Y}$, respectively. In other words, the measured input, $\tilde{u}_{\mathrm{c}}(t)$, and output, $\tilde{y}(t)$, are represented with

$$
\begin{gathered}
\tilde{u}_{\mathrm{c}}(t)=u_{\mathrm{c}}(t)+n(t) \\
\tilde{y}(t)=y(t)+v(t)
\end{gathered}
$$

where $n(t)$ and $v(t)$ are zero-mean noise signals affecting the input and output data, respectively.

Figure 11 illustrates the prediction performance of the estimated HTFs for different cases (perfect measurement, input noise only, output noise only and input and output noise) of how the noise applied to sinusoid input tests with different frequencies. Note that the addition of noise deteriorates the prediction performance of the estimated HTFs as expected. The single sinusoid excitation results show that our identification strategy is robust to noise corruption in input and output data up to SNR values of 12.5 (where $\mathrm{SNR}:=\left(A_{\text {signal }} /\right.$ $A_{\text {noise) }}{ }^{2}$, with $A$ denoting the rms amplitude) as in the case of Hwang (1997). However, further increasing the standard deviations drastically reduces the prediction performance, since noise starts to dominate the information necessary for system identification.

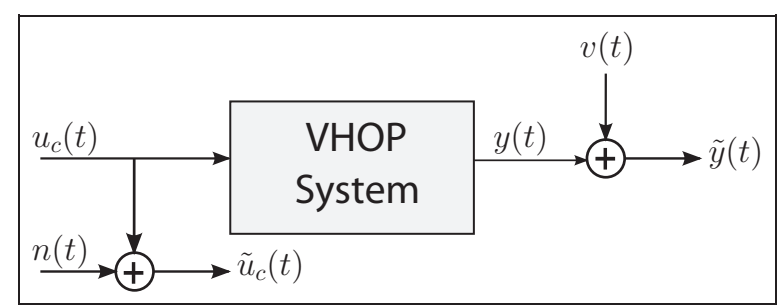

Figure 10. Block diagram representation of the VHOP system incorporating measurement noise on the input and output signals.

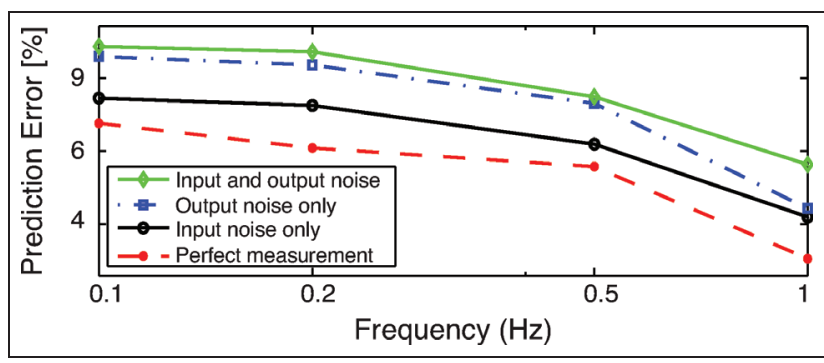

Figure I I. Percentage prediction errors $E_{\mathrm{rms}}$ for the HTF representation of the VHOP system with input and measurement noise (with SNR values of 12.5 ) in response to single sinusoid excitations at different frequencies in the range $[0, \mathrm{I}] \mathrm{Hz}$.

\section{Discussion}

\section{Summary of approach}

In this paper, we proposed a data-driven system identification strategy to represent a simple VHOP model. Most existing work on models of legged locomotion is based on explicit mathematical models. Even though these models are sufficiently accurate to describe various aspects of locomotory behaviours, their correspondence to physical behaviour degrades in the presence of unmodelled components in actual hardware platforms. Our strategy has been to use data-driven system identification techniques to describe legged locomotion systems.

The identification strategy we used relies on the perturbation of locomotion behaviours with small chirp signals, with the resulting system response used as output data. LTP system identification techniques were then applied to this inputoutput data. As a specific example, we estimated HTFs for our VHOP model around a periodic, stable hopping trajectory. We then compared the output prediction of the estimated HTFs with actual output data obtained from VHOP simulations. Our results showed that HTFs can be used as predictors of simple locomotion models on training data.

Specifically, we performed prediction tests with numerically identified HTFs on step inputs. Our results showed that the predictive performance of the HTF representation on step inputs is not as good as its performance on sinusoid inputs. Nevertheless, our results revealed that HTFs are still capable of capturing the qualitative effects of hybrid transitions associated with touchdown collisions on system output. This type of phenomena is usually difficult to incorporate into explicit 
mathematical models. We have shown that HTFs can capture and predict such discrete jumps in system dynamics, which is a promising result that highlights possible advantages of using data-driven techniques for the identification of legged locomotion models.

\section{Limitations and future work}

The hybrid nature of the VHOP model does not allow us to obtain theoretical HTFs due to discontinuities in the system state associated with collisions. A comparison of the system identification results to an ultimate, theoretical HTF representation was hence not possible. Consequently, we performed systematic simulation tests to characterize the adequacy of the numerically identified HTFs in representing system behaviour for simple legged locomotion models. To this end, we presented single sinusoid input tests to evaluate the prediction performance of HTFs. In addition, we corrupted the inputoutput data with uncorrelated noise to investigate its accuracy towards experimental inquiries. Our simulation studies showed that LTP system identification techniques yield promising results on the identification of simple locomotion models when sufficient numbers of harmonics are considered during the identification process. However, an important next step will be to more formally address hybrid transitions in a continuous HTF framework.

Our approach is based on modelling legged locomotion systems as LTP systems around their limit cycles. Legged locomotion models exhibit hybrid dynamics during their locomotion, with discrete transitions between different dynamics. For trajectories in close proximity to the limit cycle, phase resetting does not occur and these transitions can be approximated as time-dependent for stable clock-driven systems. This enables us to use an LTP structure for local system behaviour around limit cycles. We are planning to extend our methods to systems which are not clock-driven by adopting the methods developed by Ankarali and Cowan (2014) and Kiemel et al. (2013).

\section{Funding}

This material is based on work supported by National Science Foundation grants (grant numbers 0845749 and 1230493) to N. J. Cowan. This work was also supported by a grant from The Scientific and Technological Research Council of Turkey (TÜBITAK), (project number 114E277) to Uluç Saranlı. The authors thank ASELSAN Inc. and TÜBITAK for İsmail Uyanık's financial support.

\section{References}

Ankarali MM and Cowan NJ (2014) System identification of rhythmic hybrid dynamical systems via discrete time harmonic transfer functions. In: 53rd IEEE conference on decision and control (CDC'14), Los Angeles, USA, 15-17 December 2014, pp. 10171022. Piscataway: IEEE Press.

Ankarali MM and Saranli U (2010) Stride-to-stride energy regulation for robust self-stability of a torque-actuated dissipative springmass hopper. Chaos: An Interdisciplinary Journal of Nonlinear Science 20(3): 033121.
Ankarali MM and Saranli U (2011) Control of underactuated planar pronking through an embedded spring-mass hopper template. Autonomous Robots 30(2): 217-231.

Annus P, Land R, Min M and Ojarand J (2012) Simple signals for system identification. In: Mohammed Salih S (ed) Fourier Transform - Signal Processing. InTech: Rijeka, pp. 257-276.

Blickhan R and Full RJ (1993) Similarity in multilegged locomotion: Bouncing like a monopode. Journal of Comparative Physiology A: Neuroethology, Sensory, Neural, and Behavioral Physiology 173(5): 509-517.

Full RJ and Koditschek DE (1999) Templates and anchors: neuromechanical hypotheses of legged locomotion on land. Journal of Experimental Biology 202(23): 3325-3332.

Full RJ and Tu MS (1991) Mechanics of a rapid running insect: Two-, four-, and six-legged locomotion. Journal of Experimental Biology 156(1): 215-231.

Geyer H, Seyfarth A and Blickhan R (2005) Spring-mass running: Simple approximate solution and application to gait stability. Journal of Theoretical Biology 232(3): 315-328.

Guckenheimer J and Johnson S (1995) Planar hybrid systems. In: Antsaklis P, Kohn W, Nerode A and Sastry S (eds) Hybrid Systems II. New York: Springer, pp. 202-225.

Holmes P (1990) Poincaré, celestial mechanics, dynamical-systems theory and "chaos". Physics Reports 193(3): 137-163.

Hutter M, Remy CD, Hopflinger MA and Siegwart R (2010) SLIP running with an articulated robotic leg. In: IEEE/RSJ international conference on intelligent robots and systems (IROS'10), Taipei, Taiwan, 18-22 October 2010, pp. 4934-4939. Piscataway: IEEE Press.

Hwang S (1997) Frequency domain system identification of helicopter rotor dynamics incorporating models with time periodic coefficients. PhD Thesis, University of Maryland, USA.

Kiemel T, Logan D, Ivanenko Y, Lacquaniti F and Jeka JJ (2013) Characterizing perturbations of human walking in the frequency domain. In: Dynamic walking 2013, Pittsburgh, USA, 10-13 June 2013.

Leonhard A (1963) The describing function method applied for the investigation of parametric oscillations. In: 2nd world congress of the international federation of automatic control (IFAC'63), Basel, Switzerland, pp. 21-28.

Louarroudi E, Pintelon R, Lataire J and Vandersteen G (2011) Estimation of nonparametric harmonic transfer functions for linear periodically time-varying systems using periodic excitations. In: IEEE instrumentation and measurement technology conference (I2MTC), Binjiang, China, 10-12 May 2011, pp. 1-6. Piscataway: IEEE Press.

Möllerstedt E (2000) Dynamic analysis of harmonics in electrical systems. PhD Thesis, Lund Institute of Technology, Sweden.

Piovan G and Byl K (2013) Two-element control for the active SLIP model. In: IEEE international conference on robotics and automation (ICRA'13), Karlsruhe, Germany, 6-10 May, pp. 5656-5662. Piscataway: IEEE Press.

Poulakakis I and Grizzle JW (2007) Formal embedding of the spring loaded inverted pendulum in an asymmetric hopper. In: European control conference (ECC'07), Kos, Greece, 2-5 July 2007, pp. 3159-3166. Piscataway: IEEE Press.

Revzen S and Guckenheimer JM (2008) Estimating the phase of synchronized oscillators. Physical Review 78(5): 051907.

Sandberg H, Mollerstedt E and Bernhardsson (2005) Frequencydomain analysis of linear time-periodic systems. IEEE Transactions on Automatic Control 50(12): 1971-1983.

Saranlı U (2002) Dynamic locomotion with a hexapod robot. PhD Thesis, University of Michigan, USA.

Saranli U, Arslan O, Ankaralı MM and Morgül Ö (2010) Approximate analytic solutions to non-symmetric stance trajectories of the 
passive spring-loaded inverted pendulum with damping. Nonlinear Dynamics 62(4): 729-742.

Schwind WJ (1998) Spring loaded inverted pendulum running: A plant model. PhD Thesis, University of Michigan, USA.

Schwind WJ and Koditschek DE (2000) Approximating the stance map of a 2 DOF monoped runner. Journal of Nonlinear Science 10(5): 533-588.

Secer G and Saranli U (2013) Control of monopedal running through tunable damping. In: 21st signal processing and communications applications conference (SIU'13), Haspolat, Cyprus, 24-26 April 2013, pp. 1-4. Piscataway: IEEE Press.

Siddiqi A (2001) Identification of the harmonic transfer functions of a helicopter rotor. MSc Thesis, Massachusetts Institute of Technology, USA.

Sracic MW and Allen MS (2011) Method for identifying models of nonlinear systems using linear time periodic approximations. Mechanical Systems and Signal Processing 25(7): 2705-2721.
Uyanik I (2011) Adaptive control of a one-legged hopping robot through dynamically embedded spring-loaded inverted pendulum template. MSc Thesis, Bilkent University, Turkey.

Uyanik I, Morgül Ö and Saranli U (2015) Experimental validation of a feed-forward predictor for the spring-loaded inverted pendulum template. IEEE Transactions on Robotics 31(1): 208-216.

Uyanik I, Saranli U and Morgül Ö (2011) Adaptive control of a spring-mass hopper. In: IEEE international conference on robotics and automation (ICRA'11), Shanghai, China, 9-13 May, pp. 2138-2143. Piscataway: IEEE Press.

Wereley NM (1991) Analysis and control of linear periodically time varying systems. $\mathrm{PhD}$ Thesis, Massachusetts Institute of Technology, USA.

Wereley NM and Hall SR (1990) Frequency response of linear time periodic systems. In: IEEE international conference on decision and control (CDC90), vol. 6, Honolulu, USA, 5-7 December, pp. 3650-3655. Piscataway: IEEE Press. 